CANCER

\section{Priority targets}

Nat. Rev. Drug. Discov. 12, 35-50 (2013)

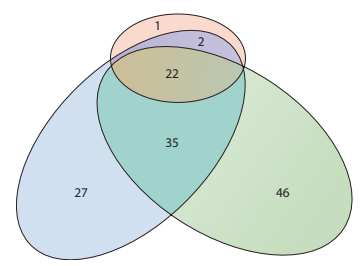

Despite the success of some molecularly targeted drugs, the selection and validation of targets suitable for drug discovery programs remains challenging. Patel et al. now report a computational approach to assess biological and chemical space for the prioritization of potential therapeutic targets and apply this approach to cancer. The approach involves the annotation of biologically relevant genes (479, in their cancer example) based on homology to targets of approved drugs, the properties of existing active molecules, three-dimensional structures, druggability, functional class, subcellular localization and other publicly available disease information. The data are then combined to rank potential targets. The authors applied their approach to propose the repurposing of drugs approved for other indications to cancer and to identify new targets. On the basis of these analyses, the authors propose that PPAR $\gamma$, DNA methyltransferase $3 \mathrm{~A}$ and aldehyde dehydrogenase - all drug targets but not indicated for use in cancer-should be evaluated for cancer. In addition, they identify 46 druggable proteins via structurebased assessments that have yet to be targeted for cancer. Although a detailed biological validation of these targets is still necessary, the new computational assessment can help scientists prioritize targets and optimize return on their monetary and labor investments. $A D$

SYNTHESIS

\section{A radical way of thinking Org. Lett. 15, 398-401 (2013)}

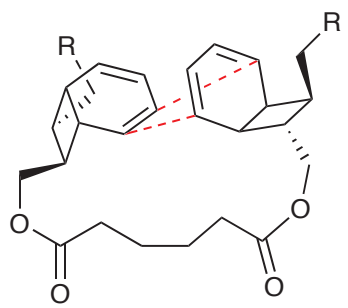

The kingianins are a class of natural products with a central scaffold that seems to include a Diels-Alder adduct between two identical or related starting materials. However, previous efforts to dimerize two kingianin A precursors were not successful, raising doubts about this potential mechanism. By taking mechanistic and stereochemical factors into account, Lim and Parker now report the successful synthesis of this intriguing pentacyclic compound. In particular, the authors suspected that a photochemical step to initiate a radical cation Diels-Alder reaction might overcome the lack of success observed previously

\section{PHOTOSYNTHESIS}

\section{Elusive reductase found}

Mol. Cell, published online 3 January 2013 doi:10.1016/j.molcel.2012.11.030
Plant photosynthesis occurs in the thylakoid membranes of chloroplasts, where light energy captured by photosystems I and II (PSI and PSII) is converted to the chemical energy of ATP and NADPH through regulated electron transport. The cyclic electron flow (CEF) pathway centers on PSI and generates proton gradients for ATP synthesis: PSI photoreduces ferredoxin (Fd) and then recovers its electrons from plastocyanin (Pc), which similarly inherits them in an ordered electron transport cascade from the cytochrome $b_{6} f$ complex (Cyt $b_{6} f$ ) and plastoquinone (PQ). Current models of CEF propose that a ferredoxin-plastoquinone reductase (FQR) must exist to complete the redox cycle between Fd and PQ. Hertle et al. now show that PGRL1, a thylakoid membraneassociated protein in Arabidopsis thaliana that was previously linked to CEF, is the missing FQR. Biochemical analyses of thylakoid membranes revealed that PGRL1 exists both as a monomer and in two dimeric forms, including a homodimer and a heterodimer with PGR5, another protein previously associated with CEF. Systematic mutagenesis of six conserved cysteine residues in PGRL1 identified three functional redox-regulated domains: a homodimerization motif that is regulated by thioredoxins, an iron-containing cofactor site and a PGR5-heterodimerization domain. In vitro assays showed that the PGRL1-PGR5 complex can accept electrons from Fd, whereas PGRL1 alone is sufficient to reduce quinones. In parallel, PGRL1 redox kinetics in planta validate that PRGL1 receives electrons from PSI in a PGR5-dependent manner. Taken together, the results support a model that PGRL1 acts as the plant FQR with the help of PGR5. using thermal conditions. The authors were further wary, however, that an uncontrolled Diels-Alder reaction could lead to multiple products, separation of which proved extremely challenging during the original isolation of the natural products. To bias the reaction toward the desired product, the authors first devised a synthetic protocol for precursor synthesis that allowed straightforward access to a single isomer. They then linked two precursors by forming esters - connected by an alkane chain - at substituents on the cyclobutane ring. Though the intramolecular reaction proceeded efficiently, the authors did observe both endo and exo products; however, these were easily separable, and the desired product could be converted in five steps to kingianin A. Given the late-stage tailoring steps, this synthesis should provide ready access to a variety of kingianin family members.

ENZYMES

\section{Specificity swap}

Glycobiology, published online 9 January 2013; doi: 10.1093/glycob/cwt003

Capsular polysaccharides-sugar chains that appear on the surface of many bacteriavary in both the carbohydrates used and the linkages between these residues. Escherichia coli $\mathrm{K} 1$ features a polysaccharide chain composed of $\alpha 2,8$-linked sialic acids, whereas that of the related $E$. coli $\mathrm{K} 92$ consists of sialic acids with alternating $\alpha 2,8$ and $\alpha 2,9$ linkages. However, each strain uses only a single enzyme, a polysialyltransferase, to construct the chains. To understand how the polysialyltransferase from K92 creates alternating linkages, Keys et al. first created a mutant library varying the six residues in the K92 transferase that are different from the $\mathrm{K} 1$ sequence within an $\mathrm{N}$-terminal region previously identified as relevant for enzyme specificity. The mutants were transformed into an engineered $E$. coli BL21 strain, in which the capsule biosynthesis genes are dormant, and the resultant polysaccharides were evaluated using linkage-specific antibodies and chemical characterization. The authors observed the formation of $\alpha 2,8$-linked structures but not $\alpha 2,9$-linked sequences, with each successful functional switch linked to a H52N mutation. Introduction of the inverse $\mathrm{N} 52 \mathrm{H}$ mutation into the K1 sequence similarly switched its specificity to that of K92's enzyme. The authors speculate that the residue controls the positioning of the polysaccharide acceptor, placing either the $\mathrm{C} 8$ or $\mathrm{C} 9$ hydroxyl group of the terminal sugar closer to the incoming donor sugar and thus determining the reaction outcome. 